\title{
Cannabis use and driving-related performance in young recreational users: a within-subject randomized clinical trial
}

\author{
Tatiana Ogourtsova PhD OT(c), Maja Kalaba MPH, Isabelle Gelinas PhD OT(c), \\ Nicol Korner-Bitensky PhD OT(c), Mark A. Ware MBBS MSc
}

Abstract

Background: With the legalization of cannabis in Canada, young adults, who are already at risk of automobile crashes, may increase their use of cannabis, which may further increase the risk of crashes. We examined the effects of inhaled cannabis on driving-related performance in healthy 18- to 24-year-old recreational cannabis users.

\begin{abstract}
Methods: In this within-subject randomized study, participants completed tests in the no-cannabis state and at 1,3 and 5 hours after inhalation of a standard 100-mg dose of cannabis. We then measured performance (in useful-field-of-view and driving-simulation tests) and self-reported perceptions (driving ability and safety, cannabis effects). Repeated-measures analysis of variance (for cannabis effects on continuous performance measures), Cochran $Q$ tests (for performance-related crash risk and binary complex simulator task scores) and correlational analyses (for self-reported perceptions relative to performance) were employed.
\end{abstract}

Results: Forty-five participants completed all 180 testing sessions. Significant effects of cannabis (relative to no cannabis) were noted on complex useful-field-of-view tasks at 3 hours (complex divided-attention task: $70 \pm 24 \mathrm{~ms}$ v. $37 \pm 12 \mathrm{~ms}$, $95 \%$ confidence intervals [Cls] 28-114 ms v. 29-45 ms, $t=-2.98$, df $=41, p=0.005$; complex selective-attention task: $102 \pm 66 \mathrm{~ms}$ v. $64 \pm 18 \mathrm{~ms}$, $95 \%$ Cls $60-144 \mathrm{~ms}$ v. $53-75 \mathrm{~ms}, t=-2.42, \mathrm{df}=41, p=0.02)$ and 5 hours (complex selective-attention task: $82 \pm 29 \mathrm{~ms}$ v. $61 \pm$ $19 \mathrm{~ms}, 95 \%$ Cls $62-100 \mathrm{~ms}$ v. 48-75 ms, $t=-2.32$, df $=41, p=0.03$ ) after cannabis use when the tasks were novel (performed in a cannabis state at the first session). Participants were significantly more likely to be classified as having a high crash risk (on the basis of simulator tasks) after cannabis use $\left(\chi^{2}=13.23\right.$, df $=1, p<0.001$, odds ratio $\left.4.31,95 \% \mathrm{Cl} 0.41-45.2\right)$ and reported significantly lower perceived driving ability and safety after cannabis use relative to non-use.

Interpretation: Among young recreational cannabis users, a 100-mg dose of cannabis by inhalation had no effect on simple drivingrelated tasks, but there was significant impairment on complex tasks, especially when these were novel. These effects, along with lower self-perceived driving ability and safety, lasted up to 5 hours after use. Trial registration: The trial was registered with Health Canada (NOL [No Objection Letter] no. 215101).

annabis is the most commonly used illicit drug globally. ${ }^{1}$ Laws legalizing possession and use of cannabis for recreational purposes ${ }^{2}$ may further increase its use. In a 2017 general population survey of 1838 current users in Canada, 36\% answered "yes" when asked "Will you consider using marijuana more often once it is legalized?" (Jason Kerr, Canadian Automobile Association, personal communication, Sept. 1, 2017). The lay public, health professionals and policy-makers have raised concern about the impact of changes in cannabis legislation on road safety. This concern is especially pronounced for young drivers, who are already known to be at high risk of crashes and who are also in the age group most likely to use cannabis. ${ }^{3}$

Previous studies have suggested that cannabis use has various effects on driving-related functions: increase in braking and choice reaction time; impairment of lane position, headway and dynamic tracking; distortion of time perception; reduction in divided and sustained attention; ${ }^{4-8}$ and increase in caution. ${ }^{9}$ What is less clear from the existing research is the time after cannabis consumption when the deficits in drivingrelated function subside (reviewed by Capler and associates ${ }^{4}$ ). Moreover, a recent literature review on driving and cannabis use (e.g., delay to driving after use, trends in cannabis use and

Competing interests: Effective July 1, 2018, Mark Ware became an employee of Canopy Growth Corporation, a Canadian licensed producer of medical cannabis. As of that date, Dr. Ware had no further involvement in analysis of the data for this study.

This article has been peer reviewed.

Correspondence to: Tatiana Ogourtsova, tatiana.ogourtsova@mail. mcgill.ca

CMAJ Open 2018. DOI:10.9778/cmajo.20180164 
driving, crash rates after legalization) was based largely on self-reported surveys and observational case-control studies of the following populations: chronic daily users, medicinal cannabis users and underage users. ${ }^{4}$ As such, the existing literature does not provide information on young healthy adults who are recreational users, and thus examination in a highquality experimental trial is warranted.

The current study aimed to answer the following questions: In young recreational cannabis users, to what extent (and for how long) is driving-related performance compromised after a usual dose of inhaled cannabis, relative to no cannabis? Are there associations between self-reported perceptions (driving ability and safety, drug effect) and performance?

\section{Methods}

\section{Study design and setting}

The study had a within-subject randomized design (with assessors blinded to time since cannabis use, and participants blinded to randomization sequence [e.g., time from cannabis use to driver-performance testing]), in which participants acted as their own controls. Time from cannabis use to testing was block-randomized into 1 of 4 sequences, such that each participant underwent 4 test sessions: without cannabis and at 1, 3 and 5 hours after cannabis use. As well, the 2 tests related to driving performance - useful field of view (UFOV) and driving simulation - were randomized, such that half of the participants began with one assessment and half began with the other. The trial took place at the Centre for Innovative Medicine, Research Institute of the McGill University Health Centre, Montréal.

\section{Population}

The target population consisted of recreational cannabis users aged 18 to 24 years. Potential participants were eligible if they met the following criteria: current recreational use of cannabis (defined as having used cannabis at least once within the past 3 months and not more than 4 times per week); held a valid driver's licence and had driven in the past 3 months; had sufficient comprehension of English or French; had sufficient cognitive ability to understand task requirements, as determined by an initial telephone interview; provided informed consent; and had a personal-use cellphone. Participants had to agree to abstain from cannabis and other illicit drugs for 48 hours before each testing day and had to be available to attend 4 testing sessions over a 4 - to 6 -week period. The following exclusion criteria were applied: medicinal cannabis use; 1 or more motor or sensory deficits of the upper or lower limb (or both) that would preclude simulator or computer use; health conditions (including seizure disorders) that might be triggered by simulator or computer use; conditions or new medications that might lead to fluctuations in health status, thus decreasing test-retest stability; pregnancy; and participation in another cannabis-related study.

We recruited participants through an online social media campaign. Those completing a preliminary eligibility screen and indicating an interest were contacted by the coordinator, who explained the purpose and procedures of the trial, determined eligibility and scheduled the first session once the participant gave verbal agreement to complete the screening process and provide written informed consent. We recruited and tested the participants between May 7 and July 5, 2018.

We based sample size calculations on performance on the primary driving-related measures, UFOV-2 and UFOV-3 (described below), ${ }^{10}$ which were the main outcomes of interest. We estimated that a sample of 36 participants would provide $95 \%$ power to detect differences of $40 \mathrm{~ms}$ (an outcome that is of clinical importance to crash risk ${ }^{11-15}$ ) in the within-person scores under the different testing conditions (e.g., various intervals from cannabis use [or no use] to driver testing). To allow for an anticipated dropout rate of $15 \%$ to $20 \%$ and to permit subgroup analyses, we aimed to enrol 50 participants.

\section{Procedures}

Each eligible, consenting participant was randomly assigned (without stratification, in blocks of 8 ) to 1 of 4 sequences on day 1 . The randomization sequence envelopes were prepared by an individual who was independent of the recruitment and data collection process. This sequence was revealed to the coordinator on the day of the first session when that person opened an opaque, numbered, sealed envelope. Participants were blinded to their sequence; they knew only that they were being tested at different times on different days. Blinding to sequence (the time to testing) was important to reduce the likelihood of systematic over- or under-consumption of cannabis by participants on days with shorter or longer delays to testing. To elucidate, if the delay was known to be relatively short (e.g., 1 h) a participant might attempt to inhale less cannabis, knowing that the testing would occur soon; conversely, a participant might attempt to inhale more on a day when driving performance was to be tested later (e.g., after $5 \mathrm{~h}$ ). Each participant underwent 4 testing sessions on separate days over a 4- to 6-week period, according to an assigned sequence:

- sequence 1: day 1 , no cannabis; day 2, testing at 1 hour after cannabis; day 3 , testing at 3 hours; day 4 , testing at 5 hours

- sequence 2: day 1 , testing at 3 hours after cannabis; day 2 , no cannabis; day 3 , testing at 5 hours; day 4 , testing at 1 hour

- sequence 3: day 1 , testing at 1 hour after cannabis; day 2 , testing at 5 hours; day 3 , no cannabis; day 4 , testing at 3 hours

- sequence 4: day 1, testing at 5 hours after cannabis; day 2, testing at 3 hours; day 3 , testing at 1 hour; day 4 , no cannabis

We chose the times from cannabis use to driver testing on the basis of consultation with experts, public health questions on time to driving after use and a review of the existing literature. Very little evidence was available on which to base timing, other than a study on resolution or near-resolution of self-reported impairments related to driving ability after 
3-4 hours in chronic high-frequency users. ${ }^{4} \mathrm{We}$ also randomly counterbalanced administration of the driving-related tasks (UFOV and simulator) within each sequence, such that half of the participants began with the UFOV. Within participants, this order remained constant.

For all sessions, participants were instructed to continue using routinely taken medications (if any) and to refrain from using cannabis, other illicit drugs and alcohol for a minimum of 48 hours before each session. We collected urine samples to verify non-use of cannabis and other illicit drugs, with analysis according to a standard procedure with high sensitivity for detection. ${ }^{16}$ A previous controlled study showed that urine testing can differentiate between recent and past use of cannabis with $80 \%$ sensitivity and $90 \%$ specificity, on the basis of a creatinine-cannabinoid normalization ratio of $0.5 .^{17}$ We incorporated this methodology into our trial by obtaining samples for urinalyses at baseline on each of the 4 test days for each participant. For female participants, we administered a urine pregnancy test. Participants were asked at each session if they had experienced any health-related changes since their previous session, and any reported changes were recorded. We proceeded with inhaled-cannabis administration and driving-related testing only if results of these tests were negative, and no health-related changes were reported.

On day 1, once the participant had signed the consent form, the following information was collected by the coordinator: basic sociodemographic characteristics (age, sex, education), driving frequency and perceived driving ability and driving safety, and cannabis-use behaviours. At each testing session, adherence with the study protocol was verified (based on questions regarding drug use, urine test and pregnancy test, as described above). After the randomization schedule revealed the procedure for the day, the identified sequence for day 1 was initiated.

On days when the participant was allocated to cannabis use, the following procedure was used. In a dedicated room with a ventilation system designed specifically for cannabis consumption via inhalation, a standard 100-mg dose of dried granulated Cannabis sativa flowers was placed into a vapourizer set at $180^{\circ} \mathrm{C}$ (Mighty Medic, Storz \& Bickel $\mathrm{GmBH}$; licensed in Canada for medical administration of cannabis on Jan. 22, 2016 [licence no. 96431]). Researchgrade herbal cannabis, with standardized levels of $12.9 \%$ (standard deviation [SD] 2.8\%) tetrahydrocannabinol (THC) and less than $1 \%$ cannabidiol, was supplied by Canopy Growth Corporation. The cannabis was chosen to reflect average THC levels in street-grade cannabis. ${ }^{2}$ The dried cannabis was prepared by the hospital pharmacy into $100-\mathrm{mg}$ doses. The participant was instructed to inhale for 5 seconds, hold the breath for 10 seconds and wait about 45 seconds between inhalations; this procedure was repeated for 5 inhalations.

Ten minutes into each session, participants completed questions regarding perceived driving ability and safety and perceived effect of the drug. After the randomly allocated wait times $(1,3$ or 5 h) or immediately (if it was a no-cannabis session), the participant underwent driving-related testing. The coordinator accompanied the participant to the driver testing room. An assessor (from a pool of 4 assessors), who was blinded to the participant's time since cannabis use, proceeded with the UFOV or simulator testing, as instructed by the coordinator (i.e., according to the randomization schedule). For all test sessions, standardized instructions were provided in French or English (as per the participant's preference). Practice trials were performed for each test, to ensure the participant's understanding of task expectations. During wait times after cannabis use, each participant remained in a private room, with access to the coordinator for any requests; meals and snacks were provided. The participant was also permitted to read, listen to music and watch movies, but not to play video games.

Three UFOV and 7 simulator tasks were completed during a 45- to 60-minute period. Breaks were introduced as needed. The order of the tasks within each test was kept constant. Between the UFOV and simulator testing, the assessor verbally presented the questions related to perceived driving ability and safety and perceived "high" that had been asked at the 10-minute point (as described above).

All 4 assessors had health care backgrounds and were trained in administration of the driving-related tests and selfreported perception questions using standard procedures. Each was blinded to the randomization schedule, and every effort was made to ensure randomization of participants across assessors, to reduce the likelihood of any single assessor seeing the same participant repeatedly.

\section{Outcome measures}

\section{Self-reported perceptions}

Perceived ability and safety to drive were measured with the following questions, with responses to the first 2 questions being based on a $10-\mathrm{cm}$ visual analogue scale (VAS): "How confident are you in your ability to drive right now?" "How confident are you in your ability to drive safely right now?" "If you were to drive right now, do you feel you would be worse, the same or better compared with when you usually drive?"

The perceived drug effect was assessed with the following question: "Compared with your usual 'high,' do you feel the same or different? If different, how?"

Participants responded verbally (responses input by the assessor on the marking sheet) or by marking the 10-cm VAS.

\section{Useful field of view (UFOV)}

The UFOV is defined as the visual field in which information can be acquired and processed; when reduced, this function has strong criterion validity in predicting crash risk. ${ }^{11}$ The UFOV test ${ }^{10}$ measures UFOV by means of 3 tasks in which the stimulus presentation ranges from very slow $(350 \mathrm{~ms})$ to extremely rapid (17 ms, which is the best possible result). In UFOV-1, a simple processing-speed task, the participant is asked to identify a centrally located object (car or truck). In UFOV-2, a more complex divided-attention task, the participant is again asked to identify whether the centrally presented 
target is a car or truck and to identify the location of a simultaneously presented peripheral target, again at different time exposures. The final and most complex selective-attention task, UFOV-3, provides a measure of distractibility by presenting the same task as the UFOV-2, this time with distractors on the screen. The UFOV provides results in milliseconds for each task, indicating the time of the stimulus presentation at which the participant is most successful (i.e., provides accurate responses).

\section{Driving simulation}

The Virage VS500M simulator (Virage Simulation Inc., Montréal) has the following components: three 55-inch $(22-\mathrm{cm})$ liquid crystal display high-resolution screens $(1920 \times 1080$ pixels), providing $180^{\circ}$ front views; 2 lateral screens positioned in the back, providing blind spot and mirror visualization; and a driving cabin equipped with automatic transmission and controls (steering wheel, pedals, dashboard). These components are mounted on a motion-vibration system that simulates acceleration, braking, pavement type and collision effects; a surround-sound system provides realistic engine sounds adjusted to various road scenarios. ${ }^{18}$ The 7 subtests measured braking reaction time; steering reaction time; braking versus steering in combined reaction time and decision accuracy; lane deviation; intersection crossing (missed crosses, crashes during crossing, duration of crossing); vigilance (accuracy and duration); and obstacle avoidance (accuracy and crash rate). ${ }^{19}$ Although the predictive validity of the simulator is less well documented than that of the UFOV, it was chosen because of evidence that simulators are associated with on-road driving, as well as strong face validity and the ability to test crash risk. ${ }^{20,21}$

\section{Statistical analysis}

To answer the main question of interest, regarding withinperson differences in driving-related performance on continuous outcomes, according to time since cannabis use relative to no cannabis, a $4 \times 4$ repeated-measures analysis of variance ${ }^{22}$ with $p<0.05$ was used, with the session sequence (1, 2, 3 or 4) and cannabis state (no use or 1,3 or $5 \mathrm{~h}$ after use) as withinsubject factors. For participants who attended all 4 sessions, we performed per-protocol analyses using SAS software, version 9.4 (SAS Institute Inc.). Residual diagnostics were conducted, and the fit of the model was ascertained. We noted the effects of sequence and cannabis state, as well as their interaction; in cases of a significant interaction, we conducted predetermined pairwise comparisons with $t$ tests. ${ }^{23}$ As such, we did not perform further post hoc analyses. We computed effect sizes using the Cohen $d$ statistic, where "small" was defined as $d \geq 0.20$, "medium" as $d \geq 0.50$ and "large" as $d \geq$ $0.80 .{ }^{24}$ In addition to the per-protocol analyses, we used single-case analyses for participants who dropped out of the study, to compare their driving-related performance with respect to the average performance of their assigned group in relation to sequence and cannabis state. We employed the Crawford and Garthwaite approach, ${ }^{25}$ which implements classical methods for comparing the score for a single case with scores obtained in a control sample. We obtained interval estimates of the effect sizes for the difference between each case and control (as normative data).

Next, we determined an overall performance-related crash risk (high or low) on the simulator, where "high" was operationally defined as intersection crossing safety below $100 \%$, obstacle avoidance crash rate above $0 \%$, vigilance accuracy below $100 \%$ and obstacle avoidance accuracy less than $100 \%$. For this dichotomous outcome, we calculated the Cochran $Q$ statistic for binary outcomes. ${ }^{26}$ This was followed by post hoc analyses with the McNemar $\chi^{2}$ test for matched pairs ${ }^{27}$ to compare no-cannabis performance with performance at various post-cannabis times (i.e., 1, 3 and $5 \mathrm{~h}$ ) and also to compare no-cannabis performance with cannabis-state performance (i.e., combined crash risk for 1, 3 and 5 h). A Bonferroni correction was applied to these analyses, and significance was accepted at $p<0.01$.

We used correlational analyses and descriptive statistics (e.g., $t$ tests, $\chi^{2}$ analysis) to explore associations between perceived driving ability, perceived driving safety, perceived "high" effect of cannabis and driving-related performance.

\section{Ethics approval}

The study was approved by McGill University Health Centre Research Ethics Board (REB no. 2018-4138). A Clinical Trial Application "No Objection Letter" (NOL no. 215101) for this trial was received from the Therapeutic Products Directorate of Health Canada.

\section{Results}

A total of 191 individuals responded to the social media recruitment campaign; of these, 126 met preliminary eligibility criteria. The coordinator contacted the first 91 people by phone: 53 (58\%) were deemed eligible following full eligibility screening, and 48 (91\%) agreed to participate, signed the informed consent form and attended the first session. Of these, $45(94 \%)$ completed the 4-session trial protocol for a total of 180 sessions (Figure 1). Four of these participants had positive urine test results on one of the trial days, and their sessions were rescheduled within the designated protocol timeframe of 4 weeks. Table 1 outlines participants' demographic characteristics, driving behaviour and typical cannabis use. Unless otherwise indicated, results are presented on the basis of these 45 participants (21 [47\%] women, mean age $20.6 \mathrm{yr}$ ).

For the participants who dropped out $(n=3)$, single-case analyses showed that overall the UFOV-2 and UFOV-3 results were not significantly different ( 7 out of 8 comparisons) from their assigned group, as per sequence and cannabis state (for details, refer to Appendix 1, available at www.cmajopen.ca/ content/6/4/E453/suppl/DC1).

When participants were asked, 10 minutes after cannabis use (via inhalation), whether the "high" they were experiencing was the same as or different from usual, 25 (56\%), 28 (62\%) and $30(67 \%)$ participants in each post-cannabis use session ( $1 \mathrm{~h}, 3 \mathrm{~h}$ and $5 \mathrm{~h}$, respectively) reported "the same." Those who reported a different experience were queried further, with "less high" being indicated by 10 (22\%), 8 (18\%) and 7 (16\%) 


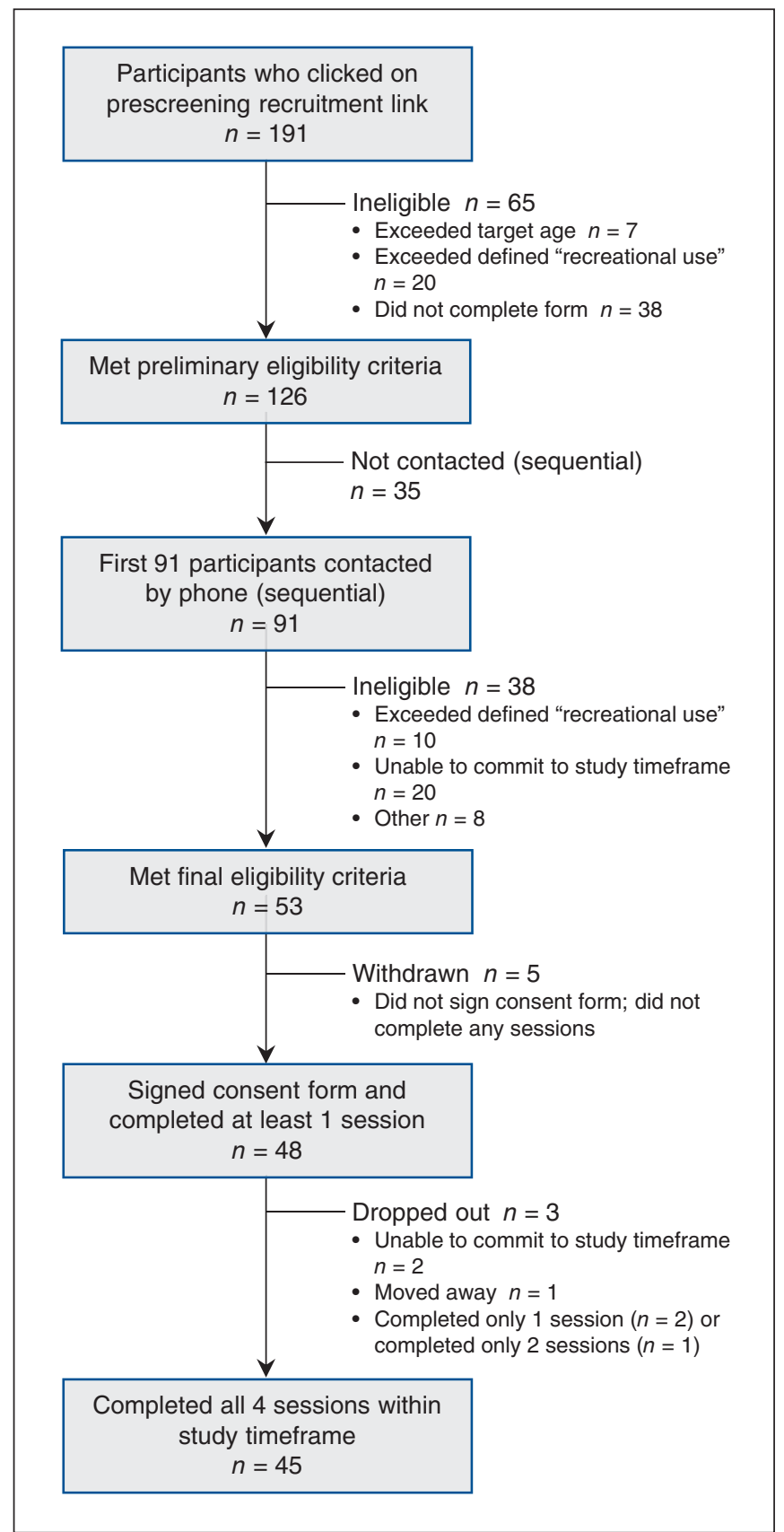

Figure 1: CONSORT diagram for a study of driving-related performance in relation to cannabis use in healthy young adults.

of participants, respectively; "more high" by 3 (7\%), 4 (9\%) and 3 (7\%), respectively; and "other" (e.g., head high, body high, tired) by $7(16 \%), 5(11 \%)$ and $5(11 \%)$, respectively.

\section{Driving-related performance}

\section{UFOV-1: simplest processing speed}

In $91 \%$ of all sessions (164/180), regardless of cannabis state, performance was at the best level possible (data presented as mean $\pm \mathrm{SD}, 95 \%$ confidence interval $[\mathrm{CI}])$ : no cannabis, $17 \pm$ $0 \mathrm{~ms} ; 1 \mathrm{~h}$ after use, $17.67 \pm 3.16 \mathrm{~ms}, 95 \%$ CI $16.88-18.46 \mathrm{~ms}$;

\begin{tabular}{|c|c|}
\hline Characteristic & $\begin{array}{c}\text { No. (\%) of participants* } \\
\qquad n=45\end{array}$ \\
\hline \multicolumn{2}{|l|}{ Demographic } \\
\hline \multicolumn{2}{|l|}{ Sex } \\
\hline Men & $24(53)$ \\
\hline Women & $21(47)$ \\
\hline Age, yr, mean $\pm S D$ & $20.6 \pm 1.3$ \\
\hline \multicolumn{2}{|l|}{ Age group, yr } \\
\hline $18-20$ & $22(49)$ \\
\hline $21-24$ & $23(51)$ \\
\hline \multicolumn{2}{|l|}{ Education completed } \\
\hline High school & $24(53)$ \\
\hline CÉGEP or university & $21(47)$ \\
\hline \multicolumn{2}{|l|}{ Driving experience } \\
\hline \multicolumn{2}{|l|}{ Duration of experience, $y r$} \\
\hline $1-3$ & $11(24)$ \\
\hline $4-6$ & $27(60)$ \\
\hline$>6$ & $7(16)$ \\
\hline \multicolumn{2}{|l|}{ Driving frequency } \\
\hline Daily or weekly & $28(62)$ \\
\hline Monthly & $11(24)$ \\
\hline Variable & $6(13)$ \\
\hline \multicolumn{2}{|l|}{ Cannabis use } \\
\hline Weekly & $17(38)$ \\
\hline Monthly & $20(44)$ \\
\hline Variable & $8(18)$ \\
\hline
\end{tabular}

$3 \mathrm{~h}$ after use, $17.31 \pm 2.09 \mathrm{~ms}, 95 \%$ CI $16.79-17.83 \mathrm{~ms}$; and $5 \mathrm{~h}$ after use, $17 \pm 0 \mathrm{~ms}$. The effects of sequence, cannabis state and the interaction of sequence $x$ cannabis state were nonsignificant $\left(\chi^{2}=2.08,2.10\right.$ and 2.10 , respectively; $\mathrm{df}=3,3$ and $5 ; p=0.6,0.6$ and 0.8$)$.

\section{UFOV-2: more complex divided-attention task}

Effects of sequence and cannabis state were both nonsignificant $(F=2.34$ and 0.10 , respectively; $\mathrm{df}=3$ for both; $p=0.09$ and $p>$ $0.9)$. However, there was a significant interaction of sequence $x$ cannabis state $(F=3.72, \mathrm{df}=9, p=0.001)$ (Figure 2). Subsequent pairwise comparisons revealed significantly worse UFOV-2 scores 3 hours after use, compared with no cannabis use, when the post-use testing was performed at the participant's first session (i.e., an unfamiliar task performed at $3 \mathrm{~h}$ after use: $70 \pm$ $24 \mathrm{~ms} \mathrm{v} .37 \pm 12 \mathrm{~ms}, 95 \%$ CI $28-114 \mathrm{~ms} \mathrm{v}$. $29-45 \mathrm{~ms}, t=-2.98$, $\mathrm{df}=41, p=0.005$ [Cohen $d=0.7$, medium effect]). Appendix 2 (available at www.cmajopen.ca/content/6/4/E453/suppl/DC1) provides average UFOV scores according to the sequence and cannabis state. 


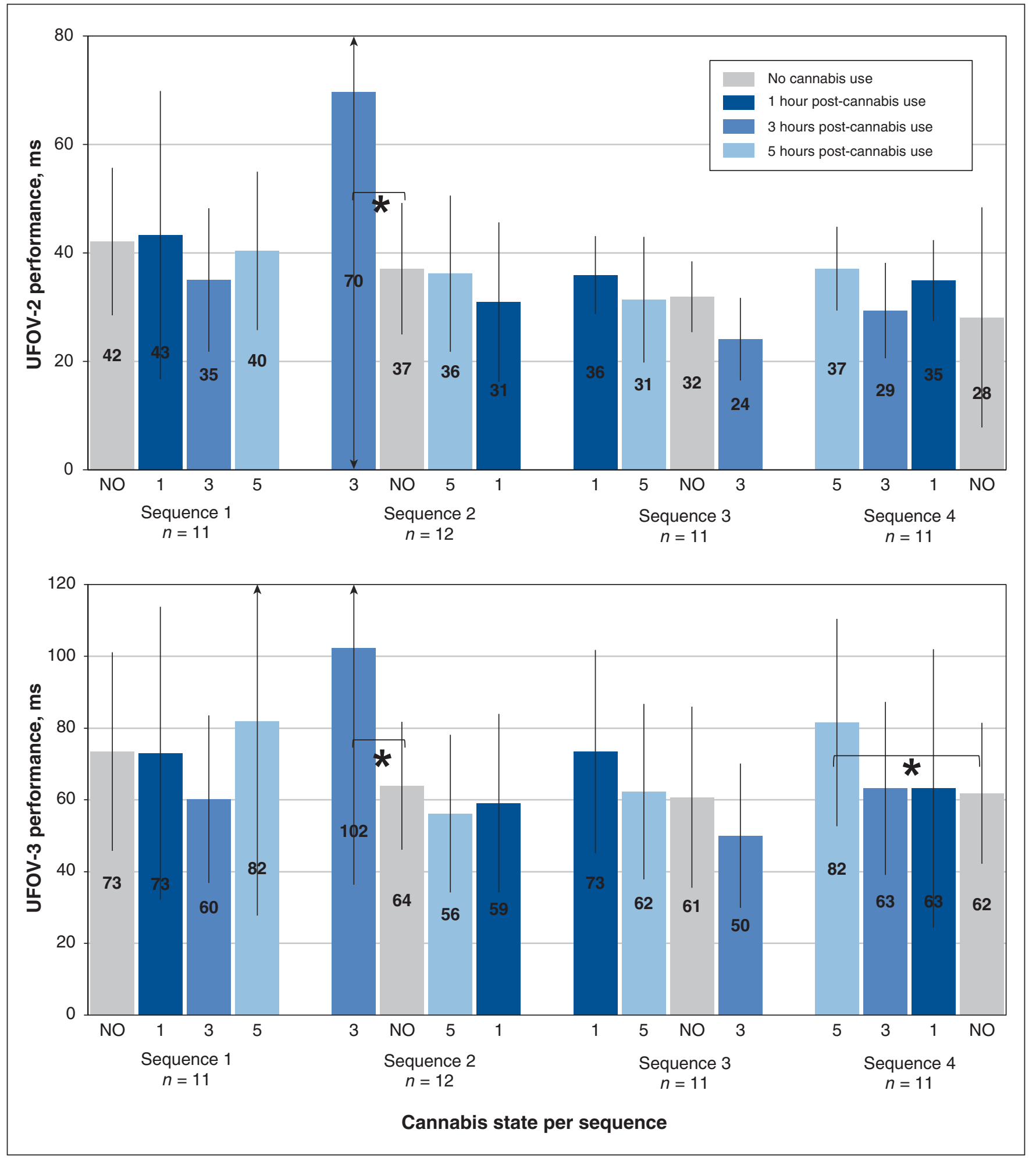

Figure 2: Performance on useful-field-of-view (UFOV) tests, according to sequence of testing and cannabis state. Performance (ms, where faster is better) was measured without cannabis and at 1,3 and 5 hours after cannabis use, according to the allocated sequence shown on horizontal axis. For UFOV-2, a significant sequence $\times$ cannabis state interaction was found $(F=3.72, \mathrm{df}=9, p=0.001)$; pairwise comparisons showed significantly worse performance at 3 hours after cannabis consumption relative to no cannabis use $(t=-2.98$, df $=41, p=0.005)$. For UFOV-3, a significant sequence $\times$ cannabis state interaction was found $(F=4.58$, df $=9, p<0.001)$; pairwise comparisons showed significantly worse performance at 3 hours after cannabis use relative to no cannabis use $(t=-2.42, \mathrm{df}=41, p=0.02)$ and at 5 hours after use $(t=-2.32, \mathrm{df}=41, p=0.03)$ relative to no use. Note: $\mathrm{NO}=$ no cannabis use; 1,3 and 5 (horizontal axis) = 1, 3 and 5 hours after cannabis use; UFOV-2 = complex divided-attention task; UFOV-3 = complex selective-attention task with distractions (see text for further description of these tests). * Significant difference at $p<0.05$. 
UFOV-3: most complex selective-attention task

The effects of sequence and cannabis state were both nonsignificant $(F=0.31$ and 0.39 , respectively; $\mathrm{df}=3$ for both; $p=0.8$ for both). However, a significant interaction of sequence $\times$ cannabis state was again found $(F=4.58, \mathrm{df}=9, p<0.001)$. Pairwise comparisons revealed significantly worse UFOV-3 scores at 3 hours after use, compared with no cannabis use, when testing was performed at the participant's first session: $102 \pm 66 \mathrm{~ms}$ v. $64 \pm 18 \mathrm{~ms}, 95 \% \mathrm{CI} 60-144 \mathrm{~ms}$ v. $53-75 \mathrm{~ms}, t=-2.42, \mathrm{df}=$ $41, p=0.02$ [Cohen $d=0.8$, large effect]). Reduced scores were also found at 5 hours after cannabis use when it was the participant's first session (i.e., unfamiliar task at $5 \mathrm{~h}$ after use: $82 \pm 29$ ms v. $61 \pm 19 \mathrm{~ms}, 95 \%$ CI $62-100 \mathrm{~ms} \mathrm{v}$. $48-75 \mathrm{~ms}, t=-2.32$, $\mathrm{df}=41, p=0.03$ [Cohen $d=0.8$, large effect]). The same pattern of poorer performance when the task was novel was noted at 1 hour after use $(73 \pm 28 \mathrm{~ms} \mathrm{v} .61 \pm 25 \mathrm{~ms}, 95 \%$ CI $54-92 \mathrm{~ms}$ v. $43-77 \mathrm{~ms})$, but the finding was not significant $(t=-1.46, \mathrm{df}=$ $41, p=0.1$ [Cohen $d=0.5$, medium effect]).

\section{Driving simulation}

Continuous responses on the simplest simulator tasks (braking, steering, lane-keeping speed control) showed no significant differences in performance after cannabis use relative to no cannabis use according to sequence $(F=1.15,1.33$ and 0.61 for braking, steering and lane-keeping speed control, respectively; $\mathrm{df}=9$ for all; $p=0.4,0.3$ and 0.8 ). There were also no significant differences in performance for the more complex tasks of intersection crossing $(F=1.71, \mathrm{df}=9, p=0.1)$, vigilance challenge accuracy $(F=0.99, \mathrm{df}=9, p=0.5)$, obstacle avoidance accuracy $(F=0.77, \mathrm{df}=9, p=0.6)$ and obstacleavoidance crash rates $(F=1.51, \mathrm{df}=9, p=0.2)$.

On the dichotomized overall outcome of high or low crash risk at each post-cannabis time point relative to no cannabis use, a twofold or greater increase in high crash-risk categorization was seen (Appendix 3, available at www.cmajopen.ca/ content/6/4/E453/suppl/DC1). On no occasion did the nocannabis state result in a greater risk of crash than the cannabis state, except on the task measuring vigilance, for which participants were twice as likely to be classified as highly vigilant at 1 hour after cannabis use. Given this trend toward a worse crash risk at 1,3 and 5 hours, further analyses contrasting driving-related performance according to the outcome cannabis state dichotomized as yes or no for cannabis use showed a significantly higher crash risk for participants after cannabis use $\left(\chi^{2}=13.23, \mathrm{df}=1, p<0.001\right.$, odds ratio [OR] 4.31, CI 0.41-45.2).

\section{Self-reported perceptions and associations}

The analyses of perceived drug effect showed that participants reporting a "usual" high 10 minutes after use had UFOV-2 and UFOV-3 scores comparable to those of participants reporting a "different" high, irrespective of the descriptors (less high, more high, other) (Figure 3).

When perceived driving ability (worse, the same or better v. usual) at 10 minutes after cannabis use was combined for the 3 test periods (1, 3 and $5 \mathrm{~h}), 23(51 \%)$ of participants thought their driving would be worse than usual, and
$22(49 \%)$ thought it would be the same as usual; none of the participants indicated that their driving would be better.

Perceived driving safety differed significantly according to time since cannabis relative to no cannabis $(F=26.01, \mathrm{df}=3$, $p<0.001$ ), such that at 1,3 and 5 hours after cannabis use, 43 (96\%), 40 (89\%) and $36(80 \%)$, respectively, scored themselves less safe to drive relative to no cannabis use. No significant associations were found between UFOV driving-related performance and perceived driving safety or ability on the continuous VAS (with the exception of UFOV-2 at $5 \mathrm{~h}$ after cannabis use [weak but significant association with perceived driving ability and safety]) (Table 2).

For the complex simulator tasks, participants who were classified as having a high crash risk after cannabis use but low crash risk when in a non-cannabis state $(n=10 / 27,37 \%)$ were significantly more likely than participants classified as low crash risk after cannabis use but high crash risk when in a non-cannabis state $(n=1 / 27,4 \%)$ to have indicated worse driving ability as compared with their usual driving ability $\left(\chi^{2}\right.$ $=7.36, \mathrm{df}=1, p=0.01$, relative risk $1.62,95 \%$ CI $1.19-2.20)$.

\section{Interpretation}

This trial was designed to determine to what extent (and for how long) driving-related performance is compromised after a usual dose of inhaled cannabis and whether there are associations between self-reported perceptions (of driving ability, driving safety and drug effect) and driving-related performance in young recreational cannabis users.

Complex driving-related performance was affected at all time points after cannabis use. Within a participant, performance was typically worse at 1,3 and 5 hours after use (relative to no use), but the result was statistically significant only for the complex tasks of UFOV-2 and UFOV-3 at 3 and 5 hours after cannabis use when the stimuli were novel (i.e., the individual had not previously experienced the task requirements). Overall, young drivers in this trial required longer stimuli presentation times to accurately respond to tasks of divided and selective attention that are known to be important predictors of crash risk. Even though this finding was not statistically significant at 1 hour after cannabis use, the medium effect size shows that performance tended to be worse at 1 hour relative to when participants were in a no-cannabis state.

On the simulator, an individual's crash risk on complex driving tasks after each cannabis use session was not significantly different from crash risk with no cannabis use. However, when the crash risk was compared in terms of performance after cannabis use (sessions combined) relative to no cannabis use, performance was almost always significantly better without cannabis. The only exception was for vigilance at 1 hour after cannabis use: vigilance was higher at that time point than for the non-cannabis state. This finding is congruent with the findings of others who have reported an increase in vigilance or caution among participants who drove after cannabis use. ${ }^{9}$

The findings on self-reported driving ability and driving safety showed that the young recreational users in this trial did not perceive themselves to be as safe to drive at 1, 3 and even 

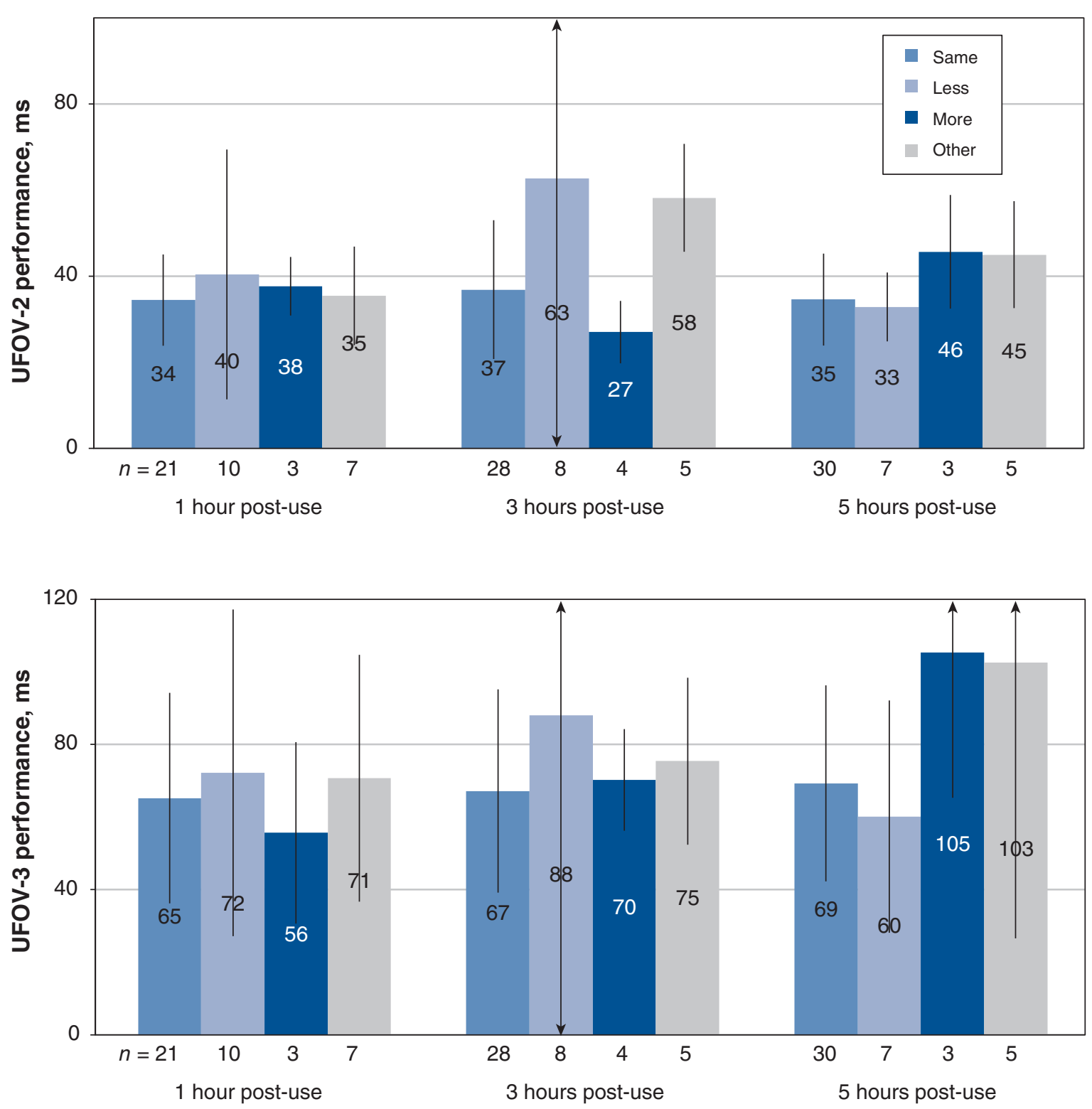

Time delay since use

Figure 3: Performance on useful-field-of-view (UFOV) tests (ms, where faster is better), according to participants' perceptions of drug effect (self-reported as "same," "less," "more" or "other"). For UFOV-2, no significant differences were found in perceived drug effects at the following sessions: 1 hour after cannabis use $(F=0.13, d f=3, p=0.9)$, 3 hours after use $(F=2.70, \mathrm{df}=3, p=0.06)$ and 5 hours after use $(F=1.81$, $\mathrm{df}=3, p=0.2$ ). For UFOV-3, no significant differences were found in perceived drug effects at the following sessions: 1 hour after cannabis use $(F=0.12, d f=3, p=0.9), 3$ hours after use $(F=0.35, \mathrm{df}=3, p=0.8)$ and 5 hours after use $(F=2.60, \mathrm{df}=3, p=0.06)$. Note: UFOV-2 $=$ complex divided-attention task; UFOV-3 = complex selective-attention task with distractions (see text for further description of these tests).

5 hours after cannabis use, relative to the no-cannabis state. This finding suggests that participants had self-awareness of their cannabis state and its potential to change their driving ability and safety. This finding will be useful to those planning self-awareness campaigns on driving safety after cannabis use. However, although trends were observed, self-perception of driving safety was not significantly correlated with poorer UFOV scores.
The measures used in the current trial each contribute to an understanding of driving performance after cannabis use. For instance, in young ${ }^{21}$ and older ${ }^{10,28}$ adults, the UFOV test has repeatedly been shown to be a strong predictor of crash rates. Specifically, a 30-ms slower score on the UFOV-3 was associated with a $10 \%$ increase in crash risk in simulated driving by young adults. ${ }^{21}$ In our study, many participants had a 


\begin{tabular}{|c|c|c|}
\hline \multirow[b]{2}{*}{ Timing and perception } & \multicolumn{2}{|c|}{ VAS measure; correlation coefficent } \\
\hline & UFOV-2 & UFOV-3 \\
\hline \multicolumn{3}{|l|}{ No cannabis use } \\
\hline Perceived driving ability & $r=0.13, p=0.4$ & $r=0.18, p=0.2$ \\
\hline Perceived driving safety & $r=0.06, p=0.7$ & $r=0.18, p=0.2$ \\
\hline \multicolumn{3}{|c|}{ At $1 \mathrm{~h}$ after cannabis use } \\
\hline Perceived driving ability & $r=-0.12, p=0.4$ & $r=-0.09, p=0.5$ \\
\hline Perceived driving safety & $r=-0.11, p=0.4$ & $r=-0.12, p=0.4$ \\
\hline \multicolumn{3}{|l|}{ At $3 \mathrm{~h}$ after cannabis use } \\
\hline Perceived driving ability & $r=0.08, p=0.6$ & $r=-0.006, p>0.9$ \\
\hline Perceived driving safety & $r=0.11, p=0.5$ & $r=0.02, p=0.8$ \\
\hline \multicolumn{3}{|l|}{ At $5 \mathrm{~h}$ after cannabis use } \\
\hline Perceived driving ability & $r=-0.40, p=0.006$ & $r=-0.005, p>0.9$ \\
\hline Perceived driving safety & $r=-0.38, p=0.009$ & $r=-0.05, p=0.7$ \\
\hline
\end{tabular}

30-ms difference in UFOV-2 and UFOV-3 scores between the no-cannabis and post-cannabis states.

These results also open the door to further inquiries (e.g., conducting studies with larger sample sizes, conducting additional simulator trials, determining usefulness of simpler driving-related tasks and benefits of current trial design). For example, this trial provides important information on the types of measures that could be used in cannabis-related driverscreening tests. To elucidate, we found that participants were particularly challenged when the tasks were presented for the first time, such that they were novel and complex. We learned that introducing novel stimuli by using randomized sequencing in a within-subject design was an important design feature. The findings also highlighted that when screening programs are launched for evaluation of drivers under the influence of cannabis, it will be important to not use testing that is familiar to the general public and to not use the same test materials repeatedly. Instead, in the development of screening tools for evaluating driver safety after cannabis use, test materials will need to be varied and novel to the user to enable high sensitivity in detecting driving performance safety issues.

As well, the ceiling effect on the simple UFOV-1 task contributes information on the validity of the testing sessions. Specifically, we had been concerned about a possible response bias, whereby participants would potentially have tried to perform more poorly in a non-cannabis state if they held the belief that cannabis use did not influence driving safety. This did not occur.

\section{Limitations}

We studied healthy young recreational users; as such, the results of this study cannot be extrapolated to daily and chronic users, nor to those with health conditions for which medicinal cannabis has been prescribed. In addition, drivingrelated response times and reactions to novel stimuli are different in young versus older individuals ${ }^{15}$ (with older adults showing age-related declines, such as reduced response time and slow response to distractors), which suggests that the differences reported here might be more pronounced in older cannabis users.

We used a standard recreational cannabis product, so the findings cannot be generalized to higher- or lower-potency products (e.g., dabbing, synthetic cannabinoids, higher-THC products).

Once cannabis use is legalized in Canada, investigating its effects on other driving outcomes that were not possible in the current trial (e.g., on-road performance) might be warranted. For analysis of simulator data, which included calculation of overall combined crash risk, the results must be interpreted with caution. However, given that there was a consistent trend toward worse performance after cannabis use relative to no cannabis use, the current findings suggest that future studies should continue to explore simulator-related performance in the hours after cannabis consumption as a useful method of detecting within-person changes in drivingrelated performance.

Our finding that performance on the UFOV was affected at 3 and 5 hours after cannabis use, but not at 1 hour after use, deserves consideration. One possibility is that during this acute post-cannabis phase, individuals are indeed able to effectively focus on tasks (e.g., findings of higher vigilance at 1 hour), but that at 3 and 5 hours after use, a different element of impairment sets in as the individual comes down from the "high" and becomes tired or more easily distracted. Another potentially related explanation is that the emergence in plasma of THC metabolites such as 11-OH THC may add to 
the drug effect. The compound 11-OH THC is a potent cannabinoid agonist and appears as inhaled THC undergoes hepatic metabolism. ${ }^{29}$ This finding deserves further study and attention. Also, plasma THC levels following cannabis use were not captured in this study, and correlations between impairment and THC levels in plasma or other body fluids were not explored. We also did not examine the effects of weight or body mass index, which might contribute to the magnitude and duration of the drug effect. Future studies should address this possibility within their designs.

The assessors, although technically blinded to cannabis state and time since use, might have been able to guess whether an individual was "high" on the basis of the objective effects of cannabis use (e.g., redness of eyes). However, all of the driving performance outcomes were recorded by the simulator and not by the assessor; as such, even if this occurred, it is unlikely to have affected the outcomes.

Finally, although per-protocol (not intention-to-treat) analyses were implemented, the performance of participants who dropped out did not differ significantly from that of their assigned group as per sequence and cannabis state.

\section{Conclusion}

We found that among young recreational cannabis users, a regular dose of cannabis had no effect on simple and learned tasks, but its use led to significant impairments on complex and novel driving-related tasks, as well as perceived driving ability and safety, for up to 5 hours after use. The present finding that the first 5 hours after cannabis use affected driving-related performance substantiates the recommendations of Canada's Lower-Risk Cannabis Use Guidelines, which recommend waiting 6 hours after cannabis use before driving. ${ }^{30}$

\section{References}

1. The bealth and social effects of nonmedical cannabis use. Geneva: World Health Organization; 2016. Available: http://www.who.int/substance_abuse/publications/ msbcannabis.pdf (accessed 2018 Sept. 4).

2. Cannabis Act, S.C. 2018 , c. 16

3. Rotermann M, Langlois K. Prevalence and correlates of marijuana use in Canada, 2012. Health Rep 2015;26(4):10-5

4. Capler R, Bilsker D, Van Pelt K, et al. Cannabis use and driving: evidence review. Burnaby (BC): Simon Fraser University, Canadian Drug Policy Coalition; 2017.

5. Ramaekers JG, Moeller MR, van Ruitenbeek P, et al. Cognition and motor control as a function of delta9-THC concentration in serum and oral fluid: limits of impairment. Drug Alcohol Depend 2006;85:114-22.

6. Riedel G, Davies SN. Cannabinoid function in learning, memory and plasticity. Handb Exp Pharmacol 2005;(168):445-77.

7. Watson TM, Mann RE. International approaches to driving under the influence of cannabis: a review of evidence on impact. Drug Alcobol Depend 2016;169:148-55.

8. Anderson BM, Rizzo M, Block RI, et al. Sex, drugs, and cognition: effects of marijuana. 7 Psychoactive Drugs 2010;42:413-24.

9. Ramaekers JG, Berghaus G, van Laar M, et al. Dose related risk of motor vehicle crashes after cannabis use. Drug Alcobol Depend 2004;73:109-19.

10. Ball K, Owsley C. The useful field of view test: a new technique for evaluating age-related declines in visual function. 7 Am Optom Assoc 1993;64:71-9.

11. Goode KT, Ball KK, Sloane M, et al. Useful field of view and other neurocognitive indicators of crash risk in older adults. 7 Clin Psychol Med Settings 1998;5:425-40.

12. Mazer BL, Sofer S, Korner-Bitensky N, et al. Use of UFOV to evaluate and retrain visual attention skills in clients with stroke. Am 7 Occup Ther 2001; 55:552-7.

13. Owsley C, Ball K, Sloane ME, et al. Visual/cognitive correlates of vehicle accidents in older drivers. Psychol Aging 1991;6:403-15.
14. Owsley C, Ball K, McGwin GJR, et al. Visual processing impairment and risk of motor vehicle crash among older adults. FAMA 1998;279:1083-8.

15. Sekuler AB, Bennett PJ, Mamelak M. Effects of aging on the useful field of view. Exp Aging Res 2000;26:103-20.

16. DrugCheck ${ }^{\circledR}$ onsite: drug testing training. Blue Earth (MN): Express Diagnostics International Inc. Available: http://drugcheck.com/wp-content/ uploads/2016/04/A_Training.pdf (accessed 2018 Sept. 4).

17. Verstraete AG. Detection time of drugs of abuse in blood, urine and oral fluid. Ther Drug Monit 2004;26:200-5.

18. Virage Simulation [homepage]. Montréal: Virage Simulation Inc. Available: https://viragesimulation.com/ (accessed 2018 Aug. 23).

19. Singh H, Barbour BM, Cox D. Driving rehabilitation as delivered by driving simulation. In: Fisher DL, Rizzo M, Caird J, et al., editors. Handbook of driving simulation for engineering, medicine and psychology. Boca Raton (FL): CRC Press; 2011.

20. Hartman RL, Brown TL, Milavetz G, et al. Cannabis effects on driving lateral control with and without alcohol. Drug Alcohol Depend 2015;154:25-37.

21. McManus B, Cox MK, Vance DE, et al. Predicting motor vehicle collisions in a driving simulator in young adults using the useful field of view assessment. Traffic Inj Prev 2015;16:818-23.

22. Gueorguieva R, Krystal JH. Move over ANOVA: progress in analyzing repeated-measures data and its reflection in papers published in the Archives of General Psychiatry. Arch Gen Psychiatry 2004;61:310-7.

23. Rice JA. Mathematical statistics and data analysis. 3rd ed. Belmont (CA): Duxbury Press; 2006.

24. Cohen J. Statistical power analysis for the behavioral sciences. 2nd ed. Hillsdale (NJ): Lawrence Erlbaum Associates; 1988:567 pp.

25. Crawford JR, Garthwaite PH. Investigation of the single case in neuropsychology: confidence limits on the abnormality of test scores and test score differences. Neuropsychologia 2002;40:1196-208.

26. Cochran WG. The comparison of percentages in matched samples. Biometrika 1950;37:256-66.

27. McNemar Q. Note on the sampling error of the difference between correlated proportions or percentages. Psychometrika 1947;12:153-7.

28. Clay OJ, Wadley VG, Edwards JD, et al. Cumulative meta-analysis of the relationship between useful field of view and driving performance in older adults: current and future implications. Optom Vis Sci 2005;82:724-31.

29. Grotenhermen F. Pharmacokinetics and pharmacodynamics of cannabinoids. Clin Pharmacokinet 2003;42:327-60.

30. Canada's lower-risk cannabis use guidelines (LRCUG). Toronto: Centre for Addiction and Mental Health. Available: https://www.camh.ca/-/media/files/lrcug professional-pdf (accessed 2018 Sept. 4).

Affiliations: School of Physical and Occupational Therapy (Ogourtsova, Gelinas, Korner-Bitensky), Faculty of Medicine, McGill University; Montreal General Hospital (Kalaba, Ware), McGill University Health Centre, Montréal, Que.

Contributors: Nicol Korner-Bitensky and Mark Ware designed the trial protocol. Maja Kalaba recruited participants and carried out data collection and analysis. Tatiana Ogourtsova carried out data collection and analysis. Nicol Korner-Bitensky carried out data analysis. Tatiana Ogourtsova, Maja Kalaba and Nicol Korner-Bitensky developed the manuscript. All of the authors critically revised the manuscript for important intellectual content, gave approval of the version to be published and agreed to be accountable for all aspects of the work.

Funding: This trial was funded by the Canadian Automobile Association.

Acknowledgements: The authors would like to acknowledge all of the trial participants; Linda Ferguson, Montreal General Hospital and McGill University Health Centre, for administrative support; Patricia McKinley, McGill University, for her contribution to the protocol; the assessor team at McGill University (Daniel Foster, Caroline Gregory and HyungMo Kang); the team at the Centre for Innovative Medicine, Research Institute of the McGill University Health Centre (Kathleen Normandin, Jasmin Mian and Anissa Capilnean, Royal Victoria Hospital pharmacy, for daily dispensing of the study drug, Carlos Guarin and Rami Tohme, Biomedical Management, for on-site technical support throughout the study, and Lynda Cedar, head, Phase 1 Unit, for clinical operations logistics and accommodations of the project); the Virage Simulation team (William Langis, Francis Lareau, Carl Frigault and Pierro Hirsch); the Storz \& Bickel team, which supplied the vapourizer devices; and the Canopy Growth Corporation team, which supplied the trial product.

Supplemental information: For reviewer comments and the original submission of this manuscript, please see www.cmajopen.ca/content/6/4/ E453/suppl/DC1. 